\title{
Profile of bosutinib and its clinical potential in the treatment of chronic myeloid leukemia
}

This article was published in the following Dove Press journal:

OncoTargets and Therapy

23 February 2013

Number of times this article has been viewed

\section{Gunhild Keller-von \\ Amsberg' \\ Steffen Koschmieder ${ }^{2}$ \\ 'Department of Hematology and Oncology, University Cancer Center Hamburg, University Hospital Hamburg Eppendorf, ${ }^{2}$ Department of Medicine (Hematology, Oncology, and Stem Cell Transplantation), University Medical Center of Aachen and RWTH Aachen University, Aachen, Germany}

Correspondence: Steffen Koschmieder Klinik für Hämatologie, Onkologie und Stammzelltransplantation, Medizinische Klinik IV,

Universitätsklinikum der RheinischWestfälischen Technischen Hochschule Aachen, Pauwelsstr 30, 52074 Aachen, Germany Tel +4924I 8089805

Fax +49 24I 8082449

Email skoschmieder@ukaachen.de

\begin{abstract}
Bosutinib (SKI-606) is an orally available, once-daily, dual Src and Abl kinase inhibitor with promising clinical potential in first-, second-, and third-line treatment of chronic myeloid leukemia (CML). Bosutinib effectively inhibits wild-type BCR-ABL and most imatinib-resistant BCR-ABL mutations except for V299L and T315I. Low hematologic toxicity is a remarkable characteristic of this novel second-generation tyrosine kinase inhibitor, and this has been ascribed to its minimal activity against the platelet-derived growth factor receptor and KIT. Low-grade, typically self-limiting diarrhea, which usually appears within the first few weeks after treatment initiation, represents the predominant toxicity of bosutinib. Other treatment-associated adverse events are mostly mild to moderate. Bosutinib has been approved by the US Food and Drug Administration for the treatment of chronic, accelerated, or blast phase Philadelphia chromosome-positive CML in adult patients with resistance or intolerance to prior therapy. This review summarizes the main properties of bosutinib and the currently available data on its clinical potential in the treatment of CML.
\end{abstract}

Keywords: bosutinib, chronic myeloid leukemia, BCR-ABL, Src/Abl kinase inhibitor, point mutation, imatinib resistance

\section{Introduction}

Chronic myeloid leukemia (CML) is a hematopoietic stem cell disorder. Reciprocal translocation of the chromosomal region carrying the proto-oncogene $c$-abl localized on chromosome 9 to the breakpoint cluster region (BCR) on chromosome 22 has been identified as the crucial step in malignant cell formation. ${ }^{1}$ The resulting BCRABL fusion gene encodes a constitutively active tyrosine kinase which significantly influences cellular signal transduction. ${ }^{2}$ Numerous preclinical and clinical studies have demonstrated that the BCR-ABL oncoprotein is the major cause of the disease, and suggest that most CML cells are oncogene-addicted to the activated kinase. The tyrosine kinase inhibitor, imatinib mesylate (formerly STI571 or CGP57148), was the first-in-class drug to inhibit BCR-ABL successfully and was approved for the treatment of CML more than ten years ago. ${ }^{3-5}$ The treatment-associated adverse effects of imatinib have been at least partially ascribed to its inhibitory activity against platelet-derived growth factor receptor, KIT, and the Abl kinases. ${ }^{6}$ The strong activity of imatinib is reflected by high rates of event-free survival and overall survival ( $81 \%$ and $85 \%$, respectively), after 8 years of follow-up within the International Randomized Study of Interferon and STI571 [IRIS] trial. ${ }^{7}$ However, after 5-8 years, only 55\%-75\% of patients were still taking imatinib within the clinical trials. One major reason aside from toxicity was the emergence of resistance, and this remains a difficult task in the 
treatment of CML. ${ }^{7,8}$ BCR-ABL kinase domain mutations impairing imatinib binding have been identified as the predominant cause of imatinib resistance. ${ }^{9}$

The second-generation tyrosine kinase inhibitors, nilotinib (formerly AMN107), dasatinib (formerly BMS354825), and bosutinib (formerly SKI-606), inhibit most resistanceconferring BCR-ABL mutations. Based on convincing results from the clinical trials, nilotinib, dasatinib, and bosutinib have been approved for the treatment of patients with CML who are resistant or intolerant to imatinib. ${ }^{10-12}$

The high efficacy of nilotinib, dasatinib, and bosutinib prompted initiation of Phase III clinical trials to evaluate the clinical potential of these three compounds in the treatment of newly diagnosed, untreated CML. In all the trials, the second-generation tyrosine kinase inhibitors were judged to be superior to imatinib with regard to molecular response rates, time to achievement of remissions, and frequency of progression to accelerated phase and/or blast crisis. ${ }^{13-15}$ However, not all of these effects were statistically significant. Moreover, due to differing clinical trial designs, in particular regarding the primary study endpoints (major molecular remission, confirmed cytogenetic remission, or complete cytogenetic remission), as well as different definitions of freedom from progression, event-free survival, and progression-free survival, the results of these trials cannot be directly compared. In this review, currently available data concerning the target profile of bosutinib and clinical potential of bosutinib are summarized.

\section{Target profile of bosutinib}

Bosutinib potently targets SRC and ABL tyrosine kinases, while activity against other kinases is distinctly lower. ${ }^{17}$ Structural and spectroscopic analyses have improved our understanding of its activity of bosutinib against imatinibresistant BCR-ABL mutants. ${ }^{16}$

\section{SRC kinase inhibition}

$\mathrm{SRC}$ is a nonreceptor protein tyrosine kinase and a member of the SRC family kinases. These kinases interact with various signal transduction pathways downstream of different surface receptors, including receptor tyrosine kinases, integrins, G protein-coupled receptors, and antigen receptors. ${ }^{17}$ SRC kinases are presumably involved in malignant cell transformation, disease progression, and metastatic spread of solid tumors. ${ }^{18-20}$ In CML, SRC family kinases have been associated with disease progression, and BCR-ABLindependent forms of imatinib resistance have been reviewed elsewhere. ${ }^{20}$ Bosutinib effectively inhibits SRC kinase at nanomolar concentrations, with an $\mathrm{IC}_{50}$ of $1.2 \mathrm{nM}$ in SRC enzymatic assay (bosutinib corresponds to compound 31a, see Table 1). ${ }^{21}$

\section{$A b l$ and $B C R-A B L$ inhibition}

The $c$ - $a b l$ gene encodes a highly conserved, ubiquitously expressed nonreceptor tyrosine kinase. ${ }^{22,23}$ Deregulation of ABL kinase activity as a result of $b c r-a b l$ translocation as well as cytoplasmic relocalization causes enhanced cellular proliferation and reduced apoptosis. ${ }^{24}$ Comparable concentrations of bosutinib and imatinib lead to decreased tyrosine phosphorylation of target proteins. In addition, both compounds inhibit the proliferation of Abelson murine leukemia virus-transformed Rat 2 fibroblasts to a similar extent. ${ }^{25}$ Conversely, the BCR-ABL shows enhanced sensitivity to bosutinib. Bosutinib virtually abrogates tyrosine phosphorylation of BCR-ABL at concentrations of $25-50 \mathrm{nM}$, whereas concentrations of $200 \mathrm{nM}$ are needed to decrease $\mathrm{v}$-Abl phosphorylation to a similar extent. ${ }^{25}$

\section{Additional targets}

More than 45 tyrosine and serine/threonine kinases have been identified as possible targets of bosutinib using chemical proteomics and in vitro kinase assays. These kinases include the apoptosis-linked STE20 kinases and CAMK2G, both of which are associated with myeloid leukemia cell proliferation. ${ }^{26}$ In addition, bosutinib reduces the activity of

Table I IC ${ }_{50}$ values for bosutinib against different kinases as determined by radioactive kinase assays using exogenous peptide substrates

\begin{tabular}{|c|c|}
\hline Protein kinase targets & Bosutinib $I C_{50}$ value $(\mathrm{nM})$ \\
\hline Fgr & $0.174 \pm 0.042$ \\
\hline Lyn & $0.850 \pm 0.247$ \\
\hline Src & 1.200 \\
\hline Abl & $2.400 \pm 0.700$ \\
\hline Csk & $314.2 \pm 78.9$ \\
\hline Syk & $4228 \pm 900$ \\
\hline Alk & $5720 \pm 1115$ \\
\hline Ret & $>20000$ \\
\hline PKA & $>20000$ \\
\hline CKI & $>20000$ \\
\hline CK2 & $>20000$ \\
\hline
\end{tabular}

Notes: Adapted with permission from Boschelli DH, Ye F, Wang YD, et al. Optimization of 4-phenylamino-3-quinolinecarbonitriles as potent inhibitors of Src kinase activity. J Med Chem. 2001;44(23):3965-3977. ${ }^{21}$ Copyright $\odot 2$ 200I American Chemical Society. Adapted from Cancer Research, Copyright @) 2006, 66(23), I I3 I4I 1322, Puttini M, Coluccia AM, Boschelli F, et al. In vitro and in vivo activity of SKI606, a novel Src-Abl inhibitor, against imatinib-resistant Bcr-Abl+ neoplastic cells, with permission from $A A C R .{ }^{28}$

Abbreviations: Fgr, Gardner-Rasheed feline sarcoma viral (v-fgr) oncogene homolog; Lyn, v-yes-I Yamaguchi sarcoma viral related oncogene homolog; Src, sarcoma oncogene; Abl, Ableson; Csk, C-src kinase; Syk, Spleen tyrosine kinase; Alk, Anaplastic lymphoma kinase; Ret, ret proto-oncogene; PKA, protein kinase A; CKI, Casein kinase 1; CK2, Casein kinase 2. 
cyclin-dependent kinase 2, which causes prominent G1 arrest of clonal hematopoietic BCR-ABL-expressing hematopoietic progenitors. ${ }^{27}$ Effects of bosutinib on different protein kinases, as evaluated by Puttini et al, are shown in Table $1 .{ }^{28}$

\section{Clinical potential of bosutinib}

Bosutinib shows high clinical potential in first-, second-, and third-line treatment of chronic phase CML. The different trials carried out for the first-, second-, and third-line settings are summarized here.

\section{Bosutinib secondary to imatinib failure}

The efficacy of bosutinib secondary to treatment failure using imatinib in chronic phase CML was evaluated in a Phase I/II, nonrandomized clinical trial. ${ }^{12}$ The study population included 288 patients with imatinib-resistant $(\mathrm{n}=200)$ or imatinib-intolerant $(\mathrm{n}=88) \mathrm{CML}$ and no other previous kinase inhibitor exposure. Resistance was defined as no hematological improvement within 4 weeks, no complete hematological response by 3 months, no cytogenetic response by 6 months, or no major cytogenetic response by 12 months with a daily imatinib dosage of at least $600 \mathrm{mg}$. Patients were considered imatinib-intolerant when grade IV hematological toxicities lasted for more than 7 days, grade 3 and 4 nonhematological toxicities appeared, grade 2 toxicities did not respond to adequate management or to dose adjustments or when a loss of a previously attained response appeared on lower-dose imatinib among patients with previous toxicity.

The primary endpoint of the study, ie, major cytogenetic response at 24 weeks, was achieved in $31 \%$ of patients $(33 \%$ in imatinib-resistant patients; $27 \%$ in imatinib-intolerant patients). ${ }^{12}$ After a median follow-up of 24.2 months, a major cytogenetic response and a complete cytogenetic response was reported in $53 \%$ and $41 \%$ of the evaluable participants, respectively. Dose intensities exceeding $350 \mathrm{mg}$ were associated with increased rates of major cytogenetic response. Most patients ( $86 \%$ ) achieved a complete hematological response, with a median time to complete hematological response of 2 weeks. Remarkably, $78 \%$ of the patients did not have a complete hematological response at the time of treatment initiation with bosutinib. Among patients with a complete cytogenetic response, $64 \%$ of imatinib-resistant and $65 \%$ of imatinib-intolerant patients achieved a major molecular response. In addition, a complete molecular response (defined as undetectable $\mathrm{BCR}-\mathrm{ABL}$, with a reverse transcriptase polymerase chain reaction sensitivity of $\geq 5 \operatorname{logs}$ ) was reported for $49 \%$ and $61 \%$ of imatinib-resistant or imatinib-intolerant patients with complete cytogenetic response, respectively.

Hematological and cytogenetic responses were examined in a subgroup of patients with known BCR-ABL mutations at the study baseline $(n=115)$. Remarkably, similar responses were observed across all $\mathrm{BCR}-\mathrm{ABL}$ mutations when compared with wild-type BCR-ABL, with the exception of the highly resistant T315I mutation. Patients with the bosutinib-resistant V299L mutation were not included in the study.

\section{Bosutinib after failure of second/ third-line therapy with dasatinib and/or nilotinib}

Safety and efficacy of bosutinib as at least third-line treatment was evaluated in a subpopulation of patients $(n=118)$ of the above described multicenter clinical trial. ${ }^{29}$ All patients had previously received imatinib and had failed secondary treatment with dasatinib and/or nilotinib. In particular, 37 were resistant and 50 were intolerant to dasatinib. An additional 27 patients were resistant to nilotinib and one exhibited nilotinib intolerance. Two patients were resistant to both dasatinib and nilotinib while one patient was intolerant to both second-generation tyrosine kinase inhibitors.

The median duration of follow-up in the study was 28.5 (0.3-56.2) months. Best cumulative cytogenetic and hematologic responses were as follows. A major cytogenetic response was reported for $31 \%$ and $30 \%$ of dasatinibresistant or dasatinib-intolerant patients, with a complete cytogenetic response in $14 \%$ and $28 \%$, respectively. A major cytogenetic response and a complete cytogenetic response were observed in $35 \%$ and $27 \%$ of individuals harboring resistance to nilotinib, respectively.

A complete hematological response was achieved or maintained by $73 \%$ of patients, including $65 \%$ of patients without a complete hematological response at baseline. In the fourth-line setting, two of three patients achieved a confirmed complete hematological response. Transformation to accelerated phase CML occurred in four third-line patients (dasatinib-resistant, $\mathrm{n}=3$; nilotinib-resistant, $\mathrm{n}=1$ ) and one fourth-line patient.

Response rates were analyzed with respect to the mutational status of BCR-ABL. The most frequent BCR-ABL mutations at baseline were F317L, T315I, G250E, and Y253H. A complete hematological response and a major cytogenetic response were observed broadly across the BCR-ABL mutants, including individuals harboring the dasatinib-resistant F317L, the nilotinib-resistant $\mathrm{Y} 253 \mathrm{H}$, 
and F359C/I/V mutations, but not T315I. Nine individuals developed new mutations in the course of bosutinib treatment (V299L, $\mathrm{n}=4$; L248V, $\mathrm{n}=2$; T315I, $\mathrm{n}=2$; F359C, $\mathrm{n}=1$; $\mathrm{G} 250 \mathrm{E}, \mathrm{n}=1$ ) and eight of these individuals discontinued bosutinib because of progressive disease or unsatisfactory response. These data are in line with the reported $\mathrm{IC}_{50}$ values for bosutinib in cell lines transfected with BCR-ABL mutants (Table 2).

\section{Bosutinib in first-line therapy of chronic phase CML}

The efficacy of bosutinib as upfront therapy for chronic phase CML was evaluated in the BELA (Bosutinib Efficacy and safety in chronic myeloid LeukemiA) trial. In this open-label, randomized, multinational, Phase III clinical trial, 502 patients were randomized to bosutinib $500 \mathrm{mg} /$ day $(\mathrm{n}=250)$ or imatinib $400 \mathrm{mg} /$ day $(\mathrm{n}=252)$ after stratification for Sokal risk score and geographic region of the centers. ${ }^{15}$

Surprisingly, the primary endpoint of the study, ie, complete cytogenetic response at 12 months, was not met because no superiority of bosutinib could be demonstrated over imatinib. In the intent-to-treat population, the rate of complete cytogenetic response at 12 months was within the same range for bosutinib-treated $(70 \%, 95 \%$ confidence interval [CI] 64-76) and imatinib-treated $(68 \%, 95 \%$ CI 62-74) patients $(P=0.601)$.

Table $2 I_{50}$ values against mutated BCR-ABL forms expressed in $\mathrm{Ba} / \mathrm{F} 3$ cells

\begin{tabular}{|c|c|c|}
\hline \multicolumn{2}{|c|}{ BCR-ABL (wild-type mutation) } & \multirow{2}{*}{$\begin{array}{l}\text { Bosutinib IC }{ }_{50} \text { value }(\mathrm{nM}) \\
41.6 \mathrm{I}\end{array}$} \\
\hline & Wild-type & \\
\hline \multicolumn{3}{|l|}{ Location of mutation } \\
\hline \multirow[t]{6}{*}{ P loop } & L248V & 147.4 \\
\hline & G250E & 179.2 \\
\hline & Q252H & 33.67 \\
\hline & Y253F & 40 \\
\hline & E255K & 394 \\
\hline & E255V & 230.1 \\
\hline \multirow[t]{2}{*}{ C helix } & D276G & 25 \\
\hline & E279K & 39.7 \\
\hline \multirow[t]{3}{*}{ ATP binding region } & V299L & 1086 \\
\hline & T3।5I & 1890 \\
\hline & F3I7L & 100.7 \\
\hline $\mathrm{SH} 2$ contact & M35IT & 29.09 \\
\hline Substrate binding region & F359V & 38.59 \\
\hline \multirow[t]{4}{*}{ A loop } & L384M & 19.54 \\
\hline & $\mathrm{H} 396 \mathrm{P}$ & 18.07 \\
\hline & H396R & 33.65 \\
\hline & G398R & 48.13 \\
\hline C terminal loop & F486S & 96.13 \\
\hline
\end{tabular}

Note: Reprinted with permission. (C) 2013, American Society of Clinical Oncology. All rights reserved. Redaelli S, Piazza R, Rostagno R, et al. Activity of bosutinib, dasatinib, and nilotinib against 18 imatinib resistant BCR/ABL mutants. J Clin Oncol. 2009;27(3):469-471..$^{55}$
In contrast, the median time to first complete cytogenetic response was achieved significantly earlier in patients treated with bosutinib (12.9 weeks, 95\% CI 12.6-13.4) when compared with imatinib (24.6 weeks, 95\% CI 24.3-25.6, $P<0.001) .{ }^{15}$

Bosutinib was also superior to imatinib concerning molecular responses. The rate of major molecular remission at 12 months was significantly higher on bosutinib (41\%, $95 \%$ CI 35-47) than on imatinib (27\%, 95\% CI 22-33, $P<0.001$ ) with MR4.0 (molecular remission defined as a $4-\log$ reduction of bcr-abl/abl or $\leq 0.01 \%$ ) (defined as BCR$\mathrm{ABL} / \mathrm{ABL} \leq 0.01)$ in $12 \%$ of bosutinib-treated patients, but in only $3 \%$ of imatinib-treated patients $(P<0.001)$. In addition, the first major molecular response was achieved significantly earlier with bosutinib (37.1 weeks, 95\% CI 36.1-48.6) than with imatinib (72.3 weeks, 95\% CI 61.1 weeks not reached, $P<0.001)$. In addition, the incidence of on-treatment progression to accelerated phase or blast crisis was lower with bosutinib $(2 \%, n=4)$ compared with imatinib $(4 \%, n=10)$, but this did not reach statistical significance. Kaplan-Meier estimates of overall survival at 12 months were greater than $99 \%$ in the bosutinib arm and $97 \%$ in the imatinib arm. ${ }^{15}$

\section{Safety and tolerability}

Bosutinib is generally well tolerated in healthy adult subjects and in CML patients. ${ }^{30}$ The hematological toxicity of bosutinib in patients with CML is manageable. As expected, similar to what was seen in other clinical trials of tyrosine kinase inhibitors in second- and third-line treatment of CML, the rate of grade $3 / 4$ hematological toxicities increases with the number of prior therapies. Grade 3/4 thrombocytopenia was observed in $14 \%$ of patients treated upfront, $25 \%$ of patients treated second-line, and $26 \%$ of patients treated third-line, while neutropenia was reported in $11 \%$ of patients treated first-line, $17 \%$ of patients treated second-line, and $20 \%$ of patients treated third-line. Worsening of anemia was less pronounced, occurring in $6 \%, 12 \%$, and $9 \%$ of patients treated first-line, second-line, and third-line, respectively. ${ }^{12,15,29}$

Grade 3/4 neutropenia in CML patients treated first-line was reduced with bosutinib as compared with imatinib (11\% versus $24 \%$, respectively) while anemia and thrombocytopenia were reported at similar frequencies in both treatment groups.

The most frequent nonhematological adverse events $(\geq 10 \%)$ of bosutinib are summarized in Table $3 .{ }^{12,15,29}$ Diarrhea was reported in up to $84 \%$ of patients. It occurred predominantly within the first 4 weeks after treatment initiation and was typically of low grade and self-limiting within a few weeks. However, up to $11 \%$ of patients developed 
Table 3 Nonhematological adverse events reported by $\geq 10 \%$ of patients with chronic myelogenous leukemia treated using bosutinib as first-, second-, or third-line therapy ${ }^{16,29}$

\begin{tabular}{|c|c|c|c|}
\hline \multirow{2}{*}{$\begin{array}{l}\text { Nonhematological } \\
\text { adverse events }\end{array}$} & \multicolumn{3}{|c|}{ All grades (only 3/4) [\%] } \\
\hline & Bosutinib first-line & Bosutinib second-line & Bosutinib third line \\
\hline Diarrhea & $68(\mathrm{II})$ & $84(9)$ & $83(8)$ \\
\hline Nausea & $31(I)$ & $44(2)$ & $46(1)$ \\
\hline Vomiting & $32(3)$ & $35(3)$ & $40(1)$ \\
\hline Rash & $20(1)$ & $34(9)$ & $27(4)$ \\
\hline Abdominal pain & II (I) & $22(I)$ & $20(1)$ \\
\hline Upper abdominal pain & $12(0)$ & na & $17(0)$ \\
\hline Fatigue & II (I) & $22(1)$ & $21(1)$ \\
\hline Pyrexia & $16(1)$ & $21(<1)$ & $14(0)$ \\
\hline Cough & $<10$ & $16(0)$ & $16(0)$ \\
\hline Headache & II (I) & $15(0)$ & $25(3)$ \\
\hline Arthralgia & $4(0)$ & $13(<1)$ & $13(1)$ \\
\hline Decreased appetite & $<10$ & $13(1)$ & $\mathrm{II}(\mathrm{I})$ \\
\hline Upper respiratory infection & $10(0)$ & $12(0)$ & $<10$ \\
\hline Constipation & $<10$ & $\mathrm{II}(<\mathrm{I})$ & II (0) \\
\hline Asthenia & $<10$ & II (2) & $<10$ \\
\hline Pruritus & $<10$ & $<10$ & $15(1)$ \\
\hline Dizziness & $<10$ & $<10$ & $12(0)$ \\
\hline Pleural effusion & $<10$ & $<10$ & II (2) \\
\hline
\end{tabular}

Note: Copyright (C) 2012. Reproduced with permission of American Society of Hematology. Khoury HJ, Cortes JE, Kantarjian HM, et al. Bosutinib is active in chronic phase chronic myeloid leukemia after imatinib and dasatinib and/or nilotinib therapy failure. Blood. 2012;1 I9(I5):3403-34I2.29

grade $3 / 4$ diarrhea. In the BELA trial, diarrhea required dose interruption or reduction in $21 \%$ and $8 \%$ of patients on bosutinib, respectively, while dose reduction of imatinib was not necessary in any of the patients, and in only $6 \%$ of these patients did imatinib have to be temporarily discontinued. ${ }^{15}$

Nonhematological laboratory abnormalities included hyperglycemia, hypermagnesemia, elevations of alanine transferase and aspartate transferase, uric acid, creatinine, lipase, and alkaline phosphatase, as well as hypocalcemia and hypophosphatemia. ${ }^{12,15,29}$ Increasing liver enzymes were associated with a significantly increased rate of treatment discontinuation on bosutinib treatment compared with imatinib in first-line treatment. Arthralgia, myalgia, muscle cramps, and bone pain, as well as peripheral or periorbital edema, were observed less frequently in bosutinib-treated patients than in imatinib-treated patients.

\section{Expert commentary}

Bosutinib shows high clinical efficacy in front-line CML therapy and were used secondary to treatment failure of imatinib, dasatinib, and/or nilotinib. Unexpectedly, despite the superiority of bosutinib compared with imatinib in in vitro and in vivo experiments, ${ }^{25,28}$ as well as encouraging data from the Phase II trial, bosutinib did not meet the primary endpoint of the BELA trial, showing a similar, but not improved, complete cytogenetic response when compared with imatinib at
12 months. ${ }^{15}$ One explanation for this nonsuperiority is the high rate of early treatment discontinuations due to diarrhea in the primary intent-to-treat study population, and these patients counted as nonresponders in the final analysis. However, other reasons for failure of bosutinib to induce higher rates of complete cytogenetic response than imatinib cannot be excluded, such as the high complete cytogenetic response rate by 12 months in the imatinib group in the BELA trial $(75 \%)$ which was higher than that in the ENESTnd (Evaluating Nilotinib Efficacy and Safety in clinical Trialsnewly diagnosed patients) and DASISION (Dasatinib versus Imatinib Study in Treatment-Naïve CML-CP Patients) studies ( $65 \%$ and $72 \%,{ }^{13,14}$ respectively), possibly due to a different distribution of risk groups in the study populations. On the other hand, the rate of major molecular response was significantly higher, and complete cytogenetic response and major molecular response were achieved more rapidly with bosutinib than with imatinib, suggesting that if early treatment failures can be reduced in the future, patients on bosutinib may achieve both complete cytogenetic response and major molecular response rates that are superior to those of imatinib.

Bosutinib is capable of overcoming the vast majority of imatinib-resistance-conferring BCR-ABL mutations, with the exception of the highly resistant T315I (resistant to imatinib, dasatinib, nilotinib, and bosutinib) and V299L mutations (resistant to dasatinib and bosutinib) as indicated 
by $\mathrm{IC}_{50}$ values of bosutinib in vitro (Table 2). In patients with omniresistant mutations, allogeneic stem cell transplantation and interferon are currently the only approved treatment options with a potential life prolongation for patients. This is of particular importance because $\mathrm{T} 315 \mathrm{I}$ is predicted to have an increased clinical impact in the future because of the growing selection pressure on resistant leukemic subclones under second-line tyrosine kinase inhibitor treatment. However, novel compounds such as the aurora kinase inhibitors, eg, danusertib (formerly PHA-739358), ${ }^{31}$ homoharringtonine, ${ }^{32}$ and ponatinib, ${ }^{33}$ successfully target the T315I mutation, and clinical trials are currently being carried out, with the first results looking very promising, as reviewed elsewhere. ${ }^{34}$ Most interestingly, treatment with ponatinib resulted in a complete hematological response in $94 \%$ and a major cytogenetic response in $63 \%$ of the patients in a Phase I clinical trial, with pretreated CML patients including individuals harboring a T315I mutation. ${ }^{35}$ These results were recently confirmed in a larger Phase II study including 449 mostly heavily pretreated chronic phase, accelerated phase, and blast crisis CML patients, as well as patients with Philadelphiapositive acute lymphoblastic leukemia with and without the T315I mutation. ${ }^{33}$

A decreased incidence of progression to accelerated phase or blast crisis has been reported for bosutinib, dasatinib, and nilotinib when compared with imatinib in upfront therapy, even though these differences have only been reported to be statistically significant in the case of nilotinib to date. ${ }^{36}$ Deep and early molecular remissions due to improved BCR-ABL binding are assumed to play a major role. In the case of bosutinib and dasatinib, one could hypothesize that inhibition of SRC may further improve disease control. Remarkably, while SRC family tyrosine kinases are of minor importance for the development of chronic phase CML, they have been suggested to be involved in progression of the disease. ${ }^{37}$ Indeed, increased expression and/or activation of Hck and Lyn were reported in CML patients progressing from chronic phase to accelerated phase or blast crisis. In addition, the presence of Lyn, Hck, and Fgr was found to be essential for the transition from chronic phase CML to lymphoid blast crisis in mice..$^{38}$ Interestingly, downregulation of Lyn expression by siRNA resulted in apoptosis of BCR-ABL-positive blasts, in particular if lymphoid in nature. ${ }^{39}$ In addition, Philadelphiapositive acute lymphoblastic leukemia developed in wildtype but not in triple knockout mice $\left(\mathrm{Lyn}^{--}, \mathrm{Hck}^{--}, \mathrm{Fgr}^{--}\right){ }^{37}$ Therefore, it is of considerable interest whether bosutinib and dasatinib will reduce the incidence of lymphoid blast crisis in patients with CML. Additionally, the impact of SRC kinase inhibition for control of lymphatic blast crisis as well as Philadelphia-positive acute lymphoblastic leukemia should be evaluated further.

The toxicity profile of bosutinib differs distinctly from the currently approved second-line and second-generation tyrosine kinase inhibitors in CML, providing an additional therapeutic means for patients with this disease. This difference may be due to the minimal activity of the dual SRC/ABL inhibitor against platelet-derived growth factor receptor and KIT, and a different inhibition profile against other target kinases. ${ }^{16,26,40,41}$ Bosutinib accounts for significantly less grade $3 / 4$ neutropenia than imatinib in the first-line setting. Interestingly, reduced toxicity of bosutinib towards normal progenitor cells has been found in vitro when compared with imatinib. ${ }^{42,43}$ In addition, the effects of bosutinib on platelet function are only marginal when compared with other tyrosine kinase inhibitors. While bleeding disorders (all grades) have been reported in $40 \%$ of dasatinib-treated patients, with $10 \%$ classified as grade $3 / 4,,^{44,45}$ hemorrhagic events were observed in only $5 \%$ of bosutinib-treated patients, and only one patient experienced grade 3 bleeding. ${ }^{12}$ Finally, fluid retention, is observed more often in patients treated with dasatinib $^{11,14,46,47}$ or imatinib ${ }^{14,48}$ than in individuals treated with bosutinib. ${ }^{12}$ Given that both bosutinib and dasatinib inhibit SRC, inhibition of SRC alone does not explain the development of dasatinib-induced pleural effusions. Similarly, the lymphocytosis seen in approximately one-third of dasatinib-treated patients, and found to carry a positive prognostic impact, was not observed in bosutinib-treated patients. ${ }^{49}$ In conclusion, due to the novel target profile of bosutinib and the high clinical potential and distinct toxicity profile, bosutinib is an important treatment alternative for patients with CML.

\section{Perspectives for the future}

The increasing number of approved tyrosine kinase inhibitors complemented by additional compounds that are in preclinical or clinical trials complicates choice of the best strategy for the individual patient in front- and second-line therapy, especially given that there are no randomized clinical trials which directly compare the second-generation tyrosine kinase inhibitors with each other. Such trials are desirable and important, but will likely never be performed. Thus, physicians' choices have to rely on clinical expertise and will take into account the relevance of potential adverse events and potential comorbidities in any given individual patient. Longer follow-up is needed to guide us during these decision processes. 
In addition, the definition of critical outcome-associated diagnostic landmarks during tyrosine kinase inhibitor treatment (likely involving the existing and potentially new polymerase chain reaction techniques) is very important in continuing to improve the survival of CML patients. ${ }^{50}$ The importance of an early major molecular response for individual life expectancy has not been finally clarified. However, Marin et al reported a significantly lower 8 -year probability of survival for patients with bcr-abl transcript levels $>9.84 \%$ at 3 months. ${ }^{51}$ In addition, decreased 5-year survival rates were found by Hanfstein et al for patients with bcr-abl levels $>10 \%$ according to the international scale at 3 months..$^{52}$ In consideration of these novel results, the upfront use of second-line tyrosine kinase inhibitors may be the preferable strategy for patients with a longer life expectancy.

Mutational analyses and comorbidities of the individual patient will be of major impact for determination of the type of second- and third-line therapy. Also, the decision and optimal timing of an allogeneic transplant in patients with CML is still a matter of ongoing debate.

An unresolved issue in the treatment of CML remains the lack of efficacy of currently available tyrosine kinase inhibitors against the quiescent stem cell pool. Indeed, alternative treatment options are required in order to eradicate minimal residual disease, eg, by induction of apoptosis of leukemic stem cells. ${ }^{42,53}$ Promising strategies include recruitment of quiescent leukemia stem cells into the cell cycle in order to sensitize leukemia stem cells to treatment with tyrosine kinase inhibitors, eg, by therapy with interferon alpha ${ }^{42,53}$ or autophagy inhibitors, as reviewed by Helgason et al. ${ }^{54}$ The steadily increasing knowledge about the biology of CML and the different drug profiles will determine the treatment strategies used for the individual patient with CML in the future.

\section{Disclosure}

SK has received travel support and speaker honoraria from, and serves on the advisory boards of Pfizer, Novartis, and Bristol-Myers Squibb. He has also received grant support from Novartis, the Novartis Foundation, and Bristol-Myers Squibb. GKA has no conflicts of interest in this work.

\section{References}

1. Rowley JD. Letter: a new consistent chromosomal abnormality in chronic myelogenous leukaemia identified by quinacrine fluorescence and Giemsa staining. Nature. 1973;243(5405):290-293.

2. Sattler M, Scheijen B, Weisberg E, Griffin JD. Mutated tyrosine kinases as therapeutic targets in myeloid leukemias. Adv Exp Med Biol. 2003;532:121-140.

3. Deininger MW, O’Brien SG, Ford JM, Druker BJ. Practical management of patients with chronic myeloid leukemia receiving imatinib. J Clin Oncol. 2003;21(8):1637-1647.
4. O'Brien SG, Guilhot F, Goldman JM, et al. International randomized study of interferon versus STI571 (IRIS) 7-year follow-up: sustained survival, low rate of transformation and increased rate of major molecular response (MMR) in patients (pts) with newly diagnosed chronic myeloid leukemia in chronic phase (CML-CP) treated with imatinib (IM). Blood. 2008;112:Abstr 186.

5. Sawyers CL, Hochhaus A, Feldman E, et al. Imatinib induces hematologic and cytogenetic responses in patients with chronic myelogenous leukemia in myeloid blast crisis: results of a Phase II study. Blood. 2002;99(10):3530-3539.

6. Buchdunger E, Zimmermann J, Mett H, et al. Inhibition of the Abl protein-tyrosine kinase in vitro and in vivo by a 2-phenylaminopyrimidine derivative. Cancer Res. 1996;56(1):100-104.

7. Deininger M, O’Brien SG, Guilhot F, et al. International randomized study of interferon vs STI571 (IRIS) 8-year follow up: sustained survival and low risk for progression or events in patients with newly diagnosed chronic myeloid leukemia in chronic phase (CML-CP) treated with imatinib. Blood. 2009;114:Abstr 1126.

8. de Lavallade H, Apperley JF, Khorashad JS, et al. Imatinib for newly diagnosed patients with chronic myeloid leukemia: incidence of sustained responses in an intention-to-treat analysis. $J$ Clin Oncol. 2008;26(20):3358-3363.

9. Ernst T, Hochhaus A. Chronic myeloid leukemia: clinical impact of BCR-ABL1 mutations and other lesions associated with disease progression. Semin Oncol. 2012;39(1):58-66.

10. Kantarjian H, Giles F, Wunderle L, et al. Nilotinib in imatinib-resistant CML and Philadelphia chromosome-positive ALL. $N$ Engl $J$ Med. 2006;354(24):2542-2551.

11. Hochhaus A, Kantarjian HM, Baccarani M, et al. Dasatinib induces notable hematologic and cytogenetic responses in chronic-phase chronic myeloid leukemia after failure of imatinib therapy. Blood. 2007;109(6):2303-2309.

12. Cortes JE, Kantarjian HM, BrummendorfTH, et al. Safety and efficacy of bosutinib (SKI-606) in chronic phase Philadelphia chromosomepositive chronic myeloid leukemia patients with resistance or intolerance to imatinib. Blood. 2011;118(17):4567-4576.

13. Saglio G, Kim DW, Issaragrisil S, et al. Nilotinib versus imatinib for newly diagnosed chronic myeloid leukemia. $N$ Engl $\mathrm{J}$ Med. 2010;362(24):2251-2259

14. Kantarjian H, Shah NP, Hochhaus A, et al. Dasatinib versus imatinib in newly diagnosed chronic-phase chronic myeloid leukemia. $N$ Engl J Med. 2010;362(24):2260-2270.

15. Cortes JE, Kim DW, Kantarjian HM, et al. Bosutinib versus imatinib in newly diagnosed chronic - phase chronic myeloid leukemia, Results from the BELA trail. J Clin Oncol. 2012;30(28):3486-3492.

16. Levinson NM, Boxer SG. Structural and spectroscopic analysis of the kinase inhibitor bosutinib and an isomer of bosutinib binding to the $\mathrm{Abl}$ tyrosine kinase domain. PLoS One. 2012;7(4):e29828.

17. Thomas SM, Brugge JS. Cellular functions regulated by Src family kinases. Annu Rev Cell Dev Biol. 1997;13:513-609.

18. Summy JM, Gallick GE. Src family kinases in tumor progression and metastasis. Cancer Metastasis Rev. 2003;22(4):337-358.

19. Johnson FM, Gallick GE. SRC family nonreceptor tyrosine kinases as molecular targets for cancer therapy. Anticancer Agents Med Chem. 2007;7(6):651-659.

20. Li S. Src-family kinases in the development and therapy of Philadelphia chromosome-positive chronic myeloid leukemia and acute lymphoblastic leukemia. Leuk Lymphoma. 2008;49(1):19-26.

21. Boschelli DH, Ye F, Wang YD, et al. Optimization of 4-phenylamino3-quinolinecarbonitriles as potent inhibitors of Src kinase activity. J Med Chem. 2001;44(23):3965-3977.

22. Laneuville P. Abl tyrosine protein kinase. Semin Immunol. 1995;7(4): 255-266.

23. Pendergast AM. Nuclear tyrosine kinases: from Abl to WEE1. Curr Opin Cell Biol. 1996;8(2):174-181.

24. Chung SW, Daniel R, Wong BY, Wong PM. The ABL genes in normal and abnormal cell development. Crit Rev Oncog. 1996;7(1-2):33-48. 
25. Golas JM, Arndt K, Etienne C, et al. SKI-606, a 4-anilino-3-quinolinecarbonitrile dual inhibitor of Src and Abl kinases, is a potent antiproliferative agent against chronic myelogenous leukemia cells in culture and causes regression of K562 xenografts in nude mice. Cancer Res. 2003;63(2):375-381.

26. Remsing Rix LL, Rix U, Colinge J, et al. Global target profile of the kinase inhibitor bosutinib in primary chronic myeloid leukemia cells. Leukemia. 2009;23(3):477-485.

27. Mancini M, Brusa G, Zuffa E, et al. Persistent Cdk2 inactivation drives growth arrest of BCR-ABL-expressing cells in response to dual inhibitor of SRC and ABL kinases SKI606. Leuk Res. 2007;31(7): 979-987.

28. Puttini M, Coluccia AM, Boschelli F, et al. In vitro and in vivo activity of SKI-606, a novel Src-Abl inhibitor, against imatinib-resistant BcrAbl+ neoplastic cells. Cancer Res. 2006;66(23):11314-11322.

29. Khoury HJ, Cortes JE, Kantarjian HM, et al. Bosutinib is active in chronic phase chronic myeloid leukemia after imatinib and dasatinib and/or nilotinib therapy failure. Blood. 2012;119(15):3403-3412.

30. Abbas R, Hug BA, Leister C, Gaaloul ME, Chalon S, Sonnichsen D. A phase I ascending single-dose study of the safety, tolerability, and pharmacokinetics of bosutinib (SKI-606) in healthy adult subjects. Cancer Chemother Pharmacol. 2012;69(1):221-227.

31. Gontarewicz A, Balabanov S, Keller G, et al. Simultaneous targeting of Aurora kinases and Bcr-Abl kinase by the small molecule inhibitor PHA-739358 is effective against imatinib-resistant BCR-ABL mutations including T315I. Blood. 2008;111(8):4355-4364.

32. Quintas-Cardama A, Cortes J. Homoharringtonine for the treatment of chronic myelogenous leukemia. Expert Opin Pharmacother. 2008;9(6):1029-1037

33. Cortes JE, Kim D-W, Pinilla-Ibarz J, et al; The PACE Study Group. Initial findings from the PACE trial: a pivotal Phase 2 study of ponatinib in patients with CML and $\mathrm{Ph}+\mathrm{ALL}$ resistant or intolerant to dasatinib or nilotinib, or with the T315I mutation. Blood. 2011;118:Abstr 109.

34. O'Hare T, Eide CA, Deininger MW. New Bcr-Abl inhibitors in chronic myeloid leukemia: keeping resistance in check. Expert Opin Investig Drugs. 2008;17(6):865-878.

35. Cortes J, Talpaz M, Bixby D, et al. A Phase 1 trial of oral ponatinib (AP24534) in patients with refractory chronic myelogenous leukemia (CML) and other hematologic malignancies: emerging safety and clinical response findings. Blood. 2010;116:Abstr 210.

36. Larson RA, Hochhaus A, Hughes TP, et al. Nilotinib vs imatinib in patients with newly diagnosed Philadelphia chromosome-positive chronic myeloid leukemia in chronic phase: ENESTnd 3-year follow-up. Leukemia. 2012;26(10):2197-2203.

37. Hu Y, Liu Y, Pelletier S, et al. Requirement of Src kinases Lyn, Hck and Fgr for BCR-ABL1-induced B-lymphoblastic leukemia but not chronic myeloid leukemia. Nat Genet. 2004;36(5):453-461.

38. Hu Y, Swerdlow S, Duffy TM, Weinmann R, Lee FY, Li S. Targeting multiple kinase pathways in leukemic progenitors and stem cells is essential for improved treatment of $\mathrm{Ph}+$ leukemia in mice. Proc Natl Acad Sci U S A. 2006;103(45):16870-16875.

39. Ptasznik A, Nakata Y, Kalota A, Emerson SG, Gewirtz AM. Short interfering RNA (siRNA) targeting the Lyn kinase induces apoptosis in primary, and drug-resistant, BCR-ABL1(+) leukemia cells. Nat Med. 2004;10(11):1187-1189.

OncoTargets and Therapy

\section{Publish your work in this journal}

OncoTargets and Therapy is an international, peer-reviewed, open access journal focusing on the pathological basis of all cancers, potential targets for therapy and treatment protocols employed to improve the management of cancer patients. The journal also focuses on the impact of management programs and new therapeutic agents and protocols on
40. Bantscheff M, Eberhard D, Abraham Y, et al. Quantitative chemical proteomics reveals mechanisms of action of clinical ABL kinase inhibitors. Nat Biotechnol. 2007;25(9):1035-1044.

41. Keller-v.Amsberg G, Brümmendorf TH. Novel aspects of therapy with the dual Src and Abl Kinase inhibitor bosutinib in chronic myeloid leukemia. Expert Rev Anticancer Ther. 2012;12(9):1121-1127.

42. Konig H, Holyoake TL, Bhatia R. Effective and selective inhibition of chronic myeloid leukemia primitive hematopoietic progenitors by the dual Src/Abl kinase inhibitor SKI-606. Blood. 2008;111(4): 2329-2338.

43. Bartolovic K, Balabanov S, Hartmann U, et al. Inhibitory effect of imatinib on normal progenitor cells in vitro. Blood. 2004;103(2):523-529.

44. Brave M, Goodman V, Kaminskas E, et al. Sprycel for chronic myeloid leukemia and Philadelphia chromosome-positive acute lymphoblastic leukemia resistant to or intolerant of imatinib mesylate. Clin Cancer Res. 2008;14(2):352-359.

45. Quintas-Cardama A, Han X, Kantarjian H, Cortes J. Tyrosine kinase inhibitor-induced platelet dysfunction in patients with chronic myeloid leukemia. Blood. 2009;114(2):261-263.

46. Talpaz M, Shah NP, Kantarjian H, et al. Dasatinib in imatinib-resistant Philadelphia chromosome-positive leukemias. $N$ Engl $\mathrm{J} \mathrm{Med.}$ 2006;354(24):2531-2541.

47. Quintas-Cardama A, Kantarjian H, O'Brien S, et al. Pleural effusion in patients with chronic myelogenous leukemia treated with dasatinib after imatinib failure. J Clin Oncol. 2007;25(25):3908-3914.

48. Gora-Tybor J, Robak T. Targeted drugs in chronic myeloid leukemia. Curr Med Chem. 2008;15(29):3036-3051.

49. Mustjoki S, Ekblom M, Arstila TP, et al. Clonal expansion of T/NKcells during tyrosine kinase inhibitor dasatinib therapy. Leukemia. 2009;23(8):1398-1405.

50. Lima L, Bernal-Mizrachi L, Saxe D, et al. Peripheral blood monitoring of chronic myeloid leukemia during treatment with imatinib, second-line agents, and beyond. Cancer. 2011;117(6):1245-1252.

51. Marin D, Ibrahim AR, Lucas C, et al. Assessment of BCR-ABL1 transcript levels at 3 months is the only requirement for predicting outcome for patients with chronic myeloid leukemia treated with tyrosine kinase inhibitors. J Clin Oncol. 2012;30(3):232-238.

52. Hanfstein B, Muller MC, Hehlmann R, et al. Early molecular and cytogenetic response is predictive for long-term progression-free and overall survival in chronic myeloid leukemia (CML). Leukemia. 2012;26(9):2096-2102.

53. Copland M, Hamilton A, Elrick LJ, et al. Dasatinib (BMS-354825) targets an earlier progenitor population than imatinib in primary CML but does not eliminate the quiescent fraction. Blood. 2006;107(11):4532-4539.

54. Helgason GV, Karvela M, Holyoake TL. Kill one bird with two stones: potential efficacy of BCR-ABL and autophagy inhibition in CML. Blood. 2011;118(8):2035-2043.

55. Redaelli S, Piazza R, Rostagno R, et al. Activity of bosutinib, dasatinib, and nilotinib against 18 imatinib resistant BCR/ABL mutants. J Clin Oncol. 2009;27(3):469-471.

patient perspectives such as quality of life, adherence and satisfaction. The manuscript management system is completely online and includes a very quick and fair peer-review system, which is all easy to use. Visit http://www.dovepress.com/testimonials.php to read real quotes from published authors. 